\title{
Nuclear microRNAs and their unconventional role in regulating non-coding RNAs
}

\author{
Hongwei Liang, Junfeng Zhang, Ke Zen, Chen-Yu Zhang ${ }^{\bowtie}$, Xi Chen ${ }^{\varpi}$ \\ Jiangsu Engineering Research Center for microRNA Biology and Biotechnology, State Key Laboratory of Pharmaceutical \\ Biotechnology, School of Life Sciences, Nanjing University, Nanjing 210093, China \\ $\triangle$ Correspondence: xichen@nju.edu.cn (X. Chen), cyzhang@nju.edu.cn (C. Zhang) \\ Received January 2, 2013 Accepted February 22, 2013
}

\begin{abstract}
MicroRNAs (miRNAs) are small non-coding RNAs (ncRNAs) that are involved in post-transcriptional gene regulation. It has long been assumed that miRNAs exert their roles only in the cytoplasm, where they recognize their target protein-coding messenger RNAs (mRNAs), and result in translational repression or target mRNA degradation. Recent studies, however, have revealed that mature miRNAs can also be transported from the cytoplasm to the nucleus and that these nuclear miRNAs can function in an unconventional manner to regulate the biogenesis and functions of ncRNAs (including miRNAs and long ncRNAs), adding a new layer of complexity to our understanding of gene regulation. In this review, we summarize recent literature on the working model of these unconventional miRNAs and speculate on their biological significance. We have every reason to believe that these novel models of miRNA function will become a major research topic in gene regulation in eukaryotes.
\end{abstract}

KEYWORDS microRNA, nuclear microRNA, non-coding RNA, nucleus, Argonaute, Exportin, Importin

\section{INTRODUCTION}

MicroRNAs (miRNAs) are a class of naturally occurring, singlestranded, small, non-coding RNAs (ncRNAs) of 22 nucleotides in length that play pivotal roles in post-transcriptional gene regulation (Ambros, 2004; Bartel, 2004). Research during the past decade has identified major factors participating in miRNA biogenesis and has established the basic principles of miRNA function. In conventional linear processing pathways, an miRNA gene is first transcribed by RNA polymerase II or RNA polymerase III into a primary miRNA transcript (pri-miRNA)
(Ambros, 2004; Bartel, 2004). The pri-miRNA is then cleaved into a precursor miRNA (pre-miRNA; 60-80 nucleotides) by the nuclear microprocessor complex formed by Drosha and DGCR8 (Ambros, 2004; Bartel, 2004). The pre-miRNA is then transported by Exportin-5 in a RanGTP-dependent manner into the cytoplasm, where it is further cleaved into a doublestranded miRNA/miRNA* duplex by Dicer (Ambros, 2004; Bartel, 2004). Finally, one strand of the duplex (mature miRNA) is incorporated into the RNA-induced silencing complex (RISC) (Ambros, 2004; Bartel, 2004). It is generally accepted that miRNAs negatively regulate protein-coding gene expression at the post-transcriptional level, by a mechanism of either translational repression or direct mRNA degradation (Ambros, 2004; Bartel, 2004). In this manner, miRNAs play crucial regulatory roles in a wide range of biological processes, including cellular proliferation and differentiation, migration, apoptosis, development and metabolism (Ambros, 2004; Bartel, 2004). In addition, altered expression of miRNAs contributes to the pathogenesis of many human diseases (Esquela-Kerscher and Slack, 2006; van Rooij and Olson, 2007; Tang et al., 2008).

Recent studies suggest that miRNAs work in a much more sophisticated way than was initially assumed. The finding that significant amounts of mature miRNAs are localized in the nucleus (Meister et al., 2004; Politz et al., 2006; Hwang et al., 2007; Foldes-Papp et al., 2009; Liao et al., 2010; Jeffries et al., 2011) raises the interesting possibility that miRNAs may function as gene regulators in the nucleus, through a mechanism other than classic post-transcriptional repression. Indeed, recent studies by our (Tang et al., 2012) and other research groups (Hansen et al., 2011; Zisoulis et al., 2012) demonstrate that some miRNAs can act in an unconventional manner to regulate the biogenesis and function of ncRNAs, including miRNAs and long ncRNAs. These intriguing discoveries lead to a novel model of miRNA function and add new complexity to the gene regulatory network. In this review, we summarize 
recent literature on these unconventional miRNAs and hypothesize about their biological significance.

\section{PRESENCE OF miRNAs IN THE NUCLEUS}

In the conventional pathway of miRNA processing, miRNAs are transported out of the nucleus by the specific nuclear transport receptor Exportin- 5 and eventually mature in the cytoplasm, where they silence target gene expression by inhibiting protein translation or degrading mRNA molecules (Ambros, 2004; Bartel, 2004). Thus, according to this canonical model, miRNAs only exert their post-transcriptional regulatory roles in the cytoplasmic compartment. However, evidence has accumulated demonstrating that certain mature miRNAs can re-enter the nucleus, and, in some cases, are even more abundant in the nucleus than in the cytoplasm (Meister et al., 2004; Politz et al., 2006; Hwang et al., 2007; Foldes-Papp et al., 2009; Liao et al., 2010; Jeffries et al., 2011).

The first miRNA identified in the cell nucleus was miR-21. Employing a Northern blotting assay, Meister et al. analyzed total RNA isolated from the nuclear and cytoplasmic fractions of HeLa cells and found that approximately $20 \%$ of mature miR-21 was located in the nucleus (Meister et al., 2004). The first visible evidence for the existence of mature miRNAs in the nucleus was provided by Politz et al. (2006), who characterized the intracellular localization of miR-206 in a rat myogenic cell line through in situ hybridization. Their results indicated that miR-206 was not only distributed throughout the cytoplasm but also concentrated in the nucleus (Politz et al., 2006). Using high-throughput profiling technology, such as microarrays and deep sequencing, researchers compared the levels of mature miRNAs in both cytoplasmic and nuclear fractions and reported that many miRNAs were located in both the cytoplasm and the nucleus (Liao et al., 2010; Jeffries et al., 2011). These results indicated that the presence of mature miRNAs in the nucleus is a general phenomenon in mammalian cells. Foldes-Papp et al. provided unequivocal proof for the transport of miRNAs from the cytoplasm to the nucleus. Applying superquencher molecular beacon (SQMB) probes, they detected single-stranded miR-122 in living cells by hybridization without immobilization (Foldes-Papp et al., 2009). They observed that the binding of fluorescent SQMB to miR-122, which initially occurred in the cytoplasm, was increased in the nucleus of human liver cells in a time-dependent manner, implying the trafficking of a cytoplasmic miRNA into the cell nucleus (Foldes-Papp et al., 2009). Perhaps the most systematic studies in this field were performed by Hwang et al., who elucidated for the first time one possible mechanism through which mature miRNAs could re-enter the cell nucleus (Hwang et al., 2007). These authors showed that human miR-29b was predominantly localized in the nucleus, whereas other miR-29 family members, such as miR-29a, were only found in the cytoplasm. More importantly, their results suggested that a distinctive hexanucleotide 3 ' terminal motif (AGUGUU) of miR-29b guided its nuclear import (Hwang et al., 2007).

\section{PRESENCE OF ARGONAUTE PROTEINS IN THE NUCLEUS}

Consistent with the observation that mature miRNAs are concentrated in the nucleus, studies have also reported the localization of Argonaute proteins in the human cell nucleus. Robb et al. first showed the presence of active Argonaute proteins in nuclear compartments (Robb et al., 2005). In addition, Weinmann et al. detected AGO2 in the nucleus of HeLa cells (Weinmann et al., 2009). Notably, it has been shown that Argonaute proteins are directly associated with nuclear miRNAs in Caenorhabditis elegans (C. elegans) (Zisoulis et al., 2012) and human cells (Hansen et al., 2011; Zisoulis et al., 2012). Because Argonaute proteins are the catalytic center of RISC, their presence in the nucleus may imply that they have biological relevance in the nucleus. We speculate that cells may transfer not only miRNAs but also miRNA effectors to the nucleus to enhance the function of miRNAs. From this point of view, Argonaute proteins may play a critical role in stabilizing miRNAs in the nucleus, and therefore only the miRNAs that are associated with Argonautes are stable and have biological function after they enter into the nucleus, whereas the non-Argonautebound miRNAs may be simply degraded in the nucleus.

\section{NUCLEAR-CYTOPLASMIC TRANSPORT OF mIRNAs AND ARGONAUTE PROTEINS}

The above findings strongly argue that there are some mechanisms in eukaryotes to guide the cytoplasmic-nuclear transport of miRNAs and Argonaute proteins. Generally, the nuclear-cytoplasmic transport of proteins and RNAs through nuclear pore complexes (NPCs) is mediated by Importins and Exportins (Kohler and Hurt, 2007; Katahira and Yoneda, 2011; Lee et al., 2011). The interactions between transport factors and cargo molecules are regulated by the small GTPase Ran (Kohler and Hurt, 2007; Katahira and Yoneda, 2011; Lee et al., 2011). In the nucleus, Ran is present in the GTP-bound form, while in the cytoplasm, it is present in the GDP-bound form. Importins and Exportins differ diametrically in the way they harness the RanGTP gradient: Importins bind their cargo at a low RanGTP level in the cytoplasm and release cargo upon binding to RanGTP in the nucleus. In contrast, Exportins recruit their cargo at high RanGTP levels in the nucleus, and the ternary RanGTP-Exportin-cargo complex passes through the NPC into the cytoplasm, where GTPase activation triggers disassembly of the export complex. Furthermore, different Importins and Exportins vary greatly in their substrate range (Kohler and Hurt, 2007; Katahira and Yoneda, 2011; Lee et al., 2011). For example, pre-miRNAs are exported to the cytoplasm primarily by Exportin-5, whereas tRNAs are exported by Exportin-t.

Castanotto et al. provided the first evidence that Exportin-1 allowed the nuclear-cytoplasmic shuttling of mature miRNAs (Castanotto et al., 2009). Furthermore, they showed that while Exportin-1 coimmunoprecipitated with nuclear proteins such as Topo2 $\alpha$ and EZH2, it also interacted with Argonaute 
family members such as Argonaute 1 (AGO1) and Argonaute 2 (AGO2) (Castanotto et al., 2009). Interestingly, Zisoulis et al. also showed that the depletion of Exportin-1 by RNA interference resulted in a reduction of the nuclear localization of Argonaute-like Gene 1 (ALG-1), the Argonaute protein in C. elegans, but did not affect total ALG-1 levels (Zisoulis et al., 2012). These two papers suggest that Exportin-1 may serve as a pore for the translocation of both miRNAs and Argonaute proteins from the cytoplasm to the nucleus. In another interesting study, Weinmann et al. showed that Importin-8 interacted with human Argonaute proteins in a Ran-dependent manner (Weinmann et al., 2009). Additionally, they found that upon Importin-8 knockdown, the localization of AGO2 was shifted from the nucleus to the cytoplasm, whereas the total amount of AGO2 remained unaffected (Weinmann et al., 2009). The results suggest that Importin-8 can influence nuclear import of AGO2 in human cells.

In summary, the molecular basis underlying the nuclear-cytoplasmic shuttling of mature miRNAs and Argonaute proteins is not fully understood. Whether Exportin-1, Importin-8 or other members of the Importin and Exportin family are recruited during nuclear import of miRNAs remains to be investigated.

\section{NUCLEAR miRNAs DIRECTLY REGULATE miRNAs}

It is accepted that miRNAs can indirectly regulate the expression and function of other miRNAs or themselves. For example, miR-20a of the miR-17-92 cluster targets the E2F family of transcription factors, while E2F factors bind directly to the promoter of the miR-17-92 cluster and activate its transcription (Sylvestre et al., 2007). In this case, miR-20a can target its own transcription factors and constitutes a feedback loop that regulates itself. Alternatively, some miRNAs can regulate the enzymes involved in the biogenesis and action of all miRNAs, thus maintaining the homeostasis of the miRNA pathway for proper cell function. For example, two miRNAs, miR162 and miR168, can direct the cleavage of Dicer-like 1 (DCL1) and Argonaute 1 (AGO1) mRNAs in plants, indicating that these miRNAs can regulate the activity of the miRNA pathway in a feedback fashion (Vaucheret et al., 2004; Mercer et al., 2009). Nevertheless, because the targets of these miRNAs are still protein-coding mRNAs, they generally belong to conventional miRNAs and can only exert indirect regulatory control on miRNA expression.

The discovery that mature miRNAs and Argonaute proteins are localized in the nucleus prompts the intriguing hypothesis that miRNAs function as gene regulators in the nucleus through a mechanism other than classic post-transcriptional repression. Recently, we broke with the conventional concept by demonstrating that mature miRNAs in the nucleus could directly target primary miRNA transcripts, thereby modulating the biogenesis and function of these miRNAs (Fig. 1A). In that study, we showed that mouse miR-709, which is predominantly located in the nucleus of various cell types, specifically bound to a 19-nucleotide miR-709 recognition element on miR-15a/16-1 primary transcripts (pri-miR-15a/16-1) and blocked the processing of pri-miR-15a/16-1 into miR-15a/16-1 precursor (pre-miR-15a/16-1), leading to the suppression of miR-15a/16-1 maturation (Tang et al., 2012). Thus, nuclear miR-709 negatively regulates miR-15a/16-1 maturation at the post-transcriptional level, specifically, at a stage after primiRNA transcription but before pre-miRNA cleavage. Moreover, because miR-15a/16-1 is a well-known miRNA cluster that induces cell apoptosis by targeting the anti-apoptotic protein Bcell lymphoma 2 (Bcl-2), nuclear miR-709 participated in the regulation of cell apoptosis through the miR-15a/16-1 pathway (Tang et al., 2012). This study demonstrates that one miRNA can directly target the primary transcripts of other miRNAs in the nucleus and control their biogenesis. In addition, this finding suggests a potential hierarchical structure among miRNAs, which means that certain miRNAs may have priority over other miRNAs and may shape the fate of downstream miRNAs. Similar to a classic hierarchical regulatory cascade, this "miRNA hierarchy system" forms another complex regulatory network with a cascade structure, which may have the potential to govern a wide variety of biological processes and to maintain cellular homeostasis.

More surprisingly, it has been shown that miRNAs themselves can be subject to miRNA control (Fig. 1A). Using crosslinking immunopurification combined with high-throughput sequencing (CLIP-seq), Zisoulis et al. produced a genome-wide map of interactions between Argonaute and target transcripts in C. elegans and found that more than 3000 transcripts had sequences that bound ALG-1 (Zisoulis et al., 2012). Unexpectedly, the let-7 primary transcript (pri-let-7) was among the RNAs targeted by ALG-1, and the ALG-1-binding site was approximately 500 nucleotides downstream of the mature let-7 sequence, toward the 3' end of the let-7 primary transcript (Zisoulis et al., 2012). They then performed RNA immunoprecipitation assays and verified that ALG-1 physically associated with let-7 primary transcripts and that the ALG-1-binding site was essential for this interaction (Zisoulis et al., 2012). Within the ALG-1-binding region of pri-let-7, they further identified a conserved mature let-7 complementary site, and they showed that the association of ALG-1 with pri-let-7 required mature let-7 miRNA because, in worms harboring a point mutation in the mature let-7 sequence, which disrupted pairing with the target site, the pri-let-7 transcripts no longer associated with ALG-1 (Zisoulis et al., 2012). Thus, mature let-7 can pair with and regulate its own primary transcript. Furthermore, they showed that the disruption of ALG-1 binding to pri-let-7 transcripts resulted in the accumulation of pri-let-7 but the reduction of mature let-7 in animals (Zisoulis et al., 2012). This result suggests a novel manner for Argonaute to promote the processing of pri-let-7 transcripts, in which the binding of ALG-1 through mature let-7 to pri-let-7 results in reduced pri-let-7 levels but increased mature let-7 levels. Finally, they showed that ALG-1 associated with pri-let-7 transcripts in nuclear fractions and that Argonaute could also bind to pri-let-7 in human cells (Zisoulis et al., 2012). This study reveals the first example of a 


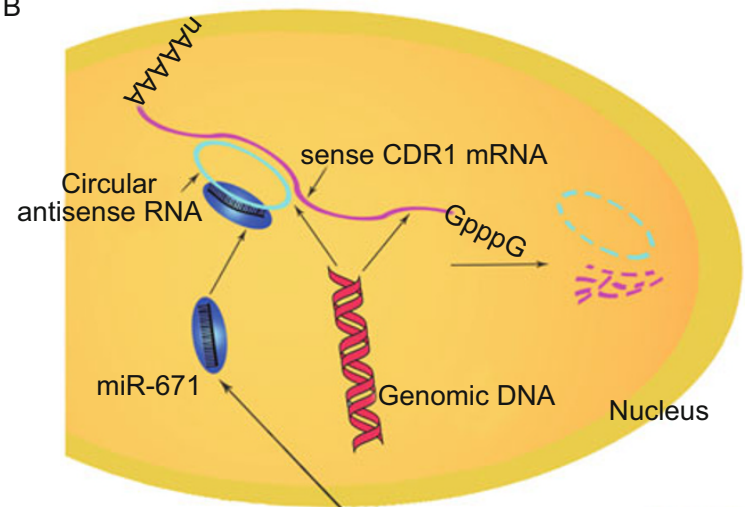

A

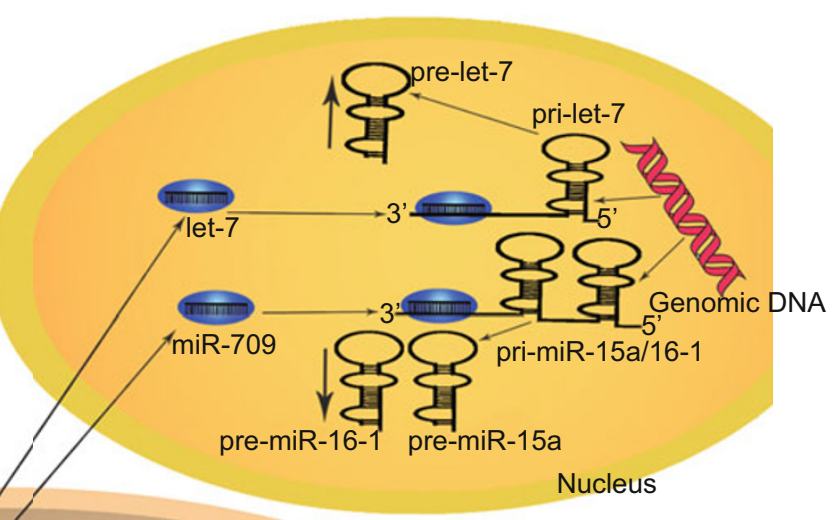

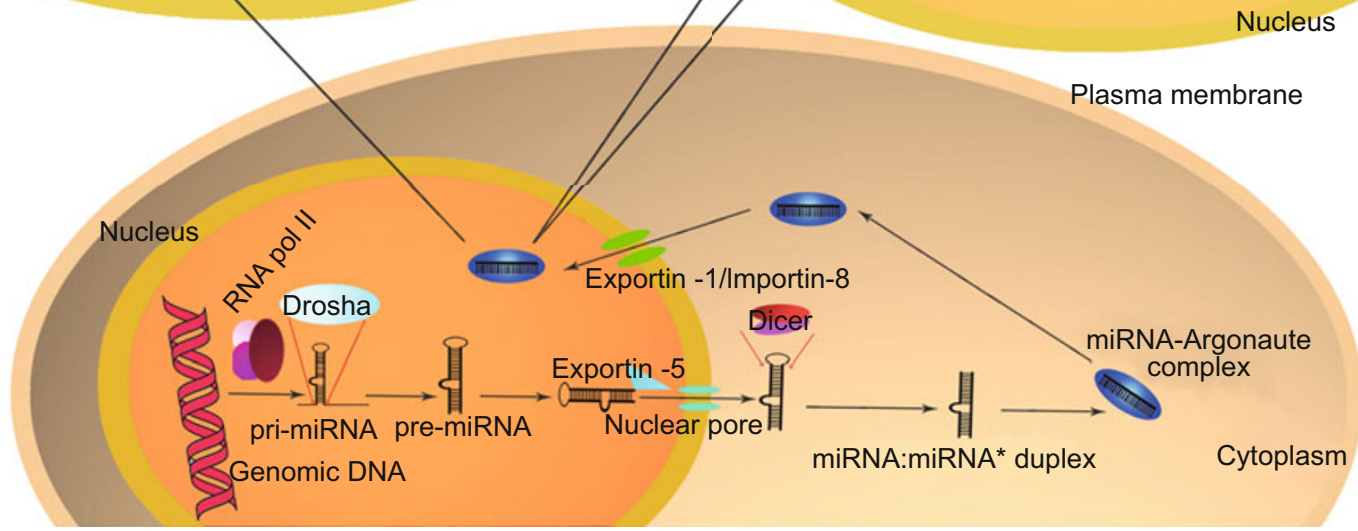

Figure 1. Unconventional models of nuclear miRNA function. According to the conventional linear processing pathway of miRNAs, mature miRNAs can only recognize sequences in protein-coding mRNAs and execute their functions predominantly in the cytoplasm. In contrast, some specific mature miRNAs (e.g., miR-709 in mouse, let-7 in C. elegans and miR-671 in humans) are preferentially imported into the nucleus rather than remaining and functioning in the cytoplasm. These miRNAs are usually combined with AGO2 proteins to form a complex, which could be imported via Exportin-1, Importin-8 or other carrier factors. (A) In the mouse nucleus, miR-709 prevents the processing of pri-miR-15a/16-1 into pre-miR-15a/16-1 via directly binding to a 19-nucleotide recognition element on pri-miR-15a/16-1 transcripts, eventually leading to the suppression of mature miRNA biogenesis. Using a similar but not identical mechanism, Argonauteassociated let-7 binds to and promotes the processing of its own primary transcript in the nucleus of $C$. elegans. (B) In the nucleus, circular CDR1 antisense RNA binds CDR1 sense mRNA and stabilizes it for export to the cytoplasm, in which the mRNA can be translated. In the presence of the miR-671-AGO2 complex in the nucleus, the antisense RNA is degraded, destabilizing the sense mRNA.

direct miRNA autoregulatory loop, in which mature let-7, with the help of Argonaute proteins, binds and promotes the processing of its own primary transcript. This amplification mechanism may be important for the efficient production of mature let-7 from the oscillating levels of primary transcript substrates during $C$. elegans development.

\section{NUCLEAR miRNAs DIRECTLY REGULATE LONG ncRNAs}

Long ncRNAs are generally considered to be non-proteincoding transcripts longer than 200 nucleotides (Mercer et al., 2009). In humans and other complex eukaryotes, most of the genome is transcribed to produce large numbers of long ncRNAs (Mercer et al., 2009). However, despite accumulating evidence suggesting that the majority of long ncRNAs are likely to be functional, only a relatively small proportion of these RNAs have been demonstrated to be biologically relevant. Likewise, there is little known about the mechanisms that control the expression of functional long ncRNAs (Mercer et al., 2009).

Similar to the indirect regulation of miRNAs by miRNAs, there have been reports of miRNAs serving as activators or repressors of the transcriptional initiation of long ncRNAs. For example, miR-29 can modulate the expression of a long ncRNA named maternally expressed gene 3 (MEG3) by acting on the DNA methyltransferase (DNMT) in the cellular methylation machinery (Braconi et al., 2011).

Recent evidence indicates that long ncRNAs may also serve as substrates for nuclear-localized miRNAs. For example, Zhao et al. compared the expression of messenger-like ncRNAs (mIRNAs) to tissue-specific miRNAs and showed that similarly to mRNAs, the expression levels of predicted mIRNA targets were significantly reduced in tissues in which a targeting miRNA was expressed (Zhao et al., 2008). The results suggest a potential network for the posttranscriptional regulation of 
ncRNAs by miRNAs. Notably, Hansen et al. presented the first experimental evidence that long ncRNAs are functional miRNA targets (Fig. 1B). They conducted a bioinformatics scan to investigate whether known miRNAs had extensive complementarity to validated ncRNAs and identified a circular ncRNA, which is the antisense transcript of the cerebellar degeneration-related protein 1 (CDR1) gene, that was nearly perfectly complementary to miR-671 (Hansen et al., 2011). Interestingly, their results demonstrated that miR-671 was one of the most enriched miRNAs in the nucleus. Furthermore, they showed that miR-671 directed cleavage of the CDR1 antisense transcript in an AGO2-dependent manner (Hansen et al., 2011). Surprisingly, the resulting downregulation of the circular antisense ncRNA was accompanied by a corresponding decrease in the CDR1 sense transcript (Hansen et al., 2011). Taken together, this study reveals the stabilization of a sense mRNA by a circular non-coding antisense RNA and the destabilization of the antisense RNA by a nuclear-localized miRNA via AGO2mediated cleavage. More importantly, this study expands the functions of the miRNA pathway in gene regulation and establishes a new model for controlling ncRNA expression.

\section{THE BIOLOGICAL RELEVANCE OF NUCLEAR miRNAs}

Although the functions of cytoplasmic miRNAs are well established, the role of nuclear miRNAs is only starting to be revealed. An intriguing idea raised previously is that the recycling of some miRNAs to the nucleus might simply be a way to sequester them from the cytoplasm and fine-tune their impact on translational regulation and mRNA stability. However, the discovery of the direct regulation of miRNAs or long ncRNAs by nuclear miRNAs and the localization of miRNA effectors, such as Argonaute proteins, in the nucleus strongly argue that we may have underestimated the potential function and physiological relevance of these nuclear miRNAs. Certain miRNAs can affect the expression level of many genes through affecting the biogenesis of other miRNAs. For example, although miR-709 does not recognize the anti-apoptotic protein Bcl-2, nuclear miR-709 has been shown to participate in the regulation of cell apoptosis through the miR-15a/16-1-Bcl-2 pathway (Tang et al., 2012). We propose that miRNAs have two different functional roles based on their intracellular localization: in the cytoplasm, they bind to protein-coding mRNA transcripts and regulate the translation of these target genes, whereas in the nucleus, miRNAs target ncRNAs and modulate their biogenesis and function.

Although a general picture of the formation and function of nuclear miRNAs has emerged, several questions remain to be addressed. What is the mechanism through which miRNAs are transported into the nucleus? Because Argonaute proteins bind miRNAs independently of sequence, they may not be involved in the selective processes of miRNAs re-entering the nucleus. Instead, individual miRNAs must convey important information allowing only these specific miRNAs to be imported into the nucleus rather than remain in the cytoplasm. Because the re-entry of miRNA into the nucleus is a prerequisite for the function of nuclear miRNAs, it is important to uncover the mechanisms controlling the cytoplasmic-nuclear shuttling of miRNAs. On the other hand, do nuclear miRNAs convey physiologically important information for cells? It is currently unclear whether the localization of miRNAs in the nucleus is dynamically altered under various physiological or pathological stimuli. Additional investigations are warranted to elucidate the clinical significance of nuclear miRNAs and its role in cellular development, differentiation, proliferation, apoptosis and signal transduction.

\section{CONCLUDING REMARKS}

The discovery of novel mechanisms of miRNA function significantly extends our understanding of miRNA-based gene regulation in eukaryotes. Specifically, miRNAs can not only regulate protein expression at the post-transcriptional level but also target ncRNAs and control their homeostasis. These unconventional roles for miRNAs open a new and exciting research field. We anticipate that exploring these unusual miRNAs will lead to new and exciting discoveries.

\section{ACKNOWLEDGEMENTS}

This work was supported by grants from the National Natural Science Foundation of China (Grant Nos. 81101330, 31271378, 81250044 and J1103512) and the Natural Science Foundation of Jiangsu Province (Nos. BK2011013 and BK2012014).

\section{ABBREVIATIONS}

AGO, Argonaute; ALG-1, Argonaute-like Gene 1; Bcl-2, B-cell lymphoma 2; CDR1, cerebellar degeneration-related protein 1; DCL1, Dicer-like 1; MEG3, maternally expressed gene 3; miRNA, microRNA; mIRNA, messenger-like ncRNA; mRNA, messenger RNA; ncRNA, non-coding RNA; pri-miRNA, primary miRNA transcript; RISC, RNAinduced silencing complex; SQMB, superquencher molecular beacon

\section{REFERENCES}

Ambros, V. (2004). The functions of animal microRNAs. Nature 431, 350-355.

Bartel, D.P. (2004). MicroRNAs: genomics, biogenesis, mechanism, and function. Cell 116, 281-297.

Braconi, C., Kogure, T., Valeri, N., Huang, N., Nuovo, G., Costinean, S., Negrini, M., Miotto, E., Croce, C.M., and Patel, T. (2011). microRNA-29 can regulate expression of the long non-coding RNA gene MEG3 in hepatocellular cancer. Oncogene 30, 4750-4756.

Castanotto, D., Lingeman, R., Riggs, A.D., and Rossi, J.J. (2009). CRM1 mediates nuclear-cytoplasmic shuttling of mature microRNAs. Proc Natl Acad Sci U S A 106, 21655-21659.

Esquela-Kerscher, A., and Slack, F.J. (2006). Oncomirs - microRNAs with a role in cancer. Nat Rev Cancer 6, 259-269.

Foldes-Papp, Z., Konig, K., Studier, H., Buckle, R., Breunig, H.G., Uchugonova, A., and Kostner, G.M. (2009). Trafficking of mature 
miRNA-122 into the nucleus of live liver cells. Curr Pharm Biotechnol 10, 569-578.

Hansen, T.B., Wiklund, E.D., Bramsen, J.B., Villadsen, S.B., Statham, A.L., Clark, S.J., and Kjems, J. (2011). miRNA-dependent gene silencing involving Ago2-mediated cleavage of a circular antisense RNA. EMBO J 30, 4414-4422.

Hwang, H.W., Wentzel, E.A., and Mendell, J.T. (2007). A hexanucleotide element directs microRNA nuclear import. Science 315, 97-100.

Jeffries, C.D., Fried, H.M., and Perkins, D.O. (2011). Nuclear and cytoplasmic localization of neural stem cell microRNAs. RNA 17, 675-686.

Katahira, J., and Yoneda, Y. (2011). Nucleocytoplasmic transport of microRNAs and related small RNAs. Traffic 12, 1468-1474.

Kohler, A., and Hurt, E. (2007). Exporting RNA from the nucleus to the cytoplasm. Nat Rev Mol Cell Biol 8, 761-773.

Lee, S.J., Jiko, C., Yamashita, E., and Tsukihara, T. (2011). Selective nuclear export mechanism of small RNAs. Curr Opin Struct Biol 21, 101-108

Liao, J.Y., Ma, L.M., Guo, Y.H., Zhang, Y.C., Zhou, H., Shao, P., Chen, Y.Q., and Qu, L.H. (2010). Deep sequencing of human nuclear and cytoplasmic small RNAs reveals an unexpectedly complex subcellular distribution of miRNAs and tRNA 3' trailers. PLoS One 5, e10563.

Meister, G., Landthaler, M., Patkaniowska, A., Dorsett, Y., Teng, G., and Tuschl, T. (2004). Human Argonaute2 mediates RNA cleavage targeted by miRNAs and siRNAs. Mol Cell 15, 185-197.

Mercer, T.R., Dinger, M.E., and Mattick, J.S. (2009). Long non-coding RNAs: insights into functions. Nat Rev Genet 10, 155-159.

Politz, J.C., Zhang, F., and Pederson, T. (2006). MicroRNA-206 colocalizes with ribosome-rich regions in both the nucleolus and cytoplasm of rat myogenic cells. Proc Natl Acad Sci U S A 103,
$18957-18962$.

Robb, G.B., Brown, K.M., Khurana, J., and Rana, T.M. (2005). Specific and potent RNAi in the nucleus of human cells. Nat Struct Mol Biol $12,133-137$.

Sylvestre, Y., De Guire, V., Querido, E., Mukhopadhyay, U.K., Bourdeau, V., Major, F., Ferbeyre, G., and Chartrand, P. (2007). An E2F/ miR-20a autoregulatory feedback loop. J Biol Chem 282, 2135-2143.

Tang, R., Li, L., Zhu, D., Hou, D., Cao, T., Gu, H., Zhang, J., Chen, J., Zhang, C.Y., and Zen, K. (2012). Mouse miRNA-709 directly regulates miRNA-15a/16-1 biogenesis at the posttranscriptional level in the nucleus: evidence for a microRNA hierarchy system. Cell Res 22, 504-515.

Tang, X., Tang, G., and Ozcan, S. (2008). Role of microRNAs in diabetes. Biochim Biophys Acta 1779, 697-701.

van Rooij, E., and Olson, E.N. (2007). MicroRNAs: powerful new regulators of heart disease and provocative therapeutic targets. $\mathrm{J}$ Clin Invest 117, 2369-2376.

Vaucheret, H., Vazquez, F., Crete, P., and Bartel, D.P. (2004). The action of ARGONAUTE1 in the miRNA pathway and its regulation by the miRNA pathway are crucial for plant development. Genes Dev 18, 1187-1197.

Weinmann, L., Hock, J., Ivacevic, T., Ohrt, T., Mutze, J., Schwille, P., Kremmer, E., Benes, V., Urlaub, H., and Meister, G. (2009). Importin 8 is a gene silencing factor that targets argonaute proteins to distinct mRNAs. Cell 136, 496-507.

Zhao, Y., He, S., Liu, C., Ru, S., Zhao, H., Yang, Z., Yang, P., Yuan, X., Sun, S., Bu, D., et al. (2008). MicroRNA regulation of messengerlike noncoding RNAs: a network of mutual microRNA control. Trends Genet 24, 323-327.

Zisoulis, D.G., Kai, Z.S., Chang, R.K., and Pasquinelli, A.E. (2012). Autoregulation of microRNA biogenesis by let-7 and Argonaute. Nature 486, 541-544. 